\title{
Asymmetries in the top sector
}

\author{
J.D. Morris* \\ Queen Mary University of London \\ E-mail: john.morris@cern.ch
}

An experimental overview of top-pair event asymmetries is reported, focusing on the forwardbackward asymmetry, $A_{F B}$, at the Tevatron and the charge asymmetry, $A_{c}$, at the LHC. Comparisons between $A_{F B}$ and $A_{c}$ at different ranges of $m_{t \bar{t}}$ are sensitive to various Beyond the Stand Model (BSM) models.

An overview of the first $\mathrm{CP}$ violation measurements using top-pair events is reported from both CMS and ATLAS via two different techniques that have a bright future.

In this $\sqrt{s}=8 \mathrm{TeV}$ review, all results are compatible with the Standard Model and no BSM models are ruled out. The majority of LHC results are limited in precision by their statistical uncertainty, which hints at exciting opportunities for $\sqrt{s}=13 \mathrm{TeV}$ future results.

9th International Workshop on the CKM Unitarity Triangle

28 November - 3 December 2016

Tata Institute for Fundamental Research (TIFR), Mumbai, India

${ }^{*}$ Speaker. 


\section{Forward-backward asymmetries at the Tevatron}

The Tevatron is a $p-\bar{p}$ machine with an asymmetric initial state. A forward-backward (FB) asymmetry for top-quark pair events is defined by the rapidity, $y$, of the top- and antitop-quarks, where $\Delta y=y_{t}-y_{\bar{t}}$, where $N$ is the event multiplicity, is expressed in Eq. 1.1 with the latest results [1] from the D0 and CDF experiments are shown in Figure 1a:

$$
A_{F B}=\frac{N(\Delta y>0)-N(\Delta y<0)}{N(\Delta y>0)+N(\Delta y<0)} .
$$

\section{Charge asymmetries at the $\mathrm{LHC}$}

The LHC is a $p-p$ machine with a symmetric initial state and as such it is not possible to define $A_{F B}$. Valence quarks typically have larger momentum than sea anti-quarks, and the topquark is predicted to have a larger $|y|$ than the antitop-quark. The charge asymmetry is defined in Eq. 2.1:

$$
A_{c}=\frac{N(\Delta|y|>0)-N(\Delta|y|<0)}{N(\Delta|y|>0)+N(\Delta|y|<0)}
$$

The asymmetry arises through the $q \bar{q} \rightarrow t \bar{t}$ process, however at the LHC the $g g \rightarrow t \bar{t}$ process dominates and has a diluting effect on $A_{c}$. Both the ATLAS and CMS experiments have measured $A_{c}$ in the dilepton [2,3] and $\ell+$ jets $[4,5]$ channels. Neutrinos are involved in all final states and a variety of methodologies are used to fully reconstruct the top-quark pair event from the observed detector objects. This is technically challenging and involves a probability of misassigning the (anti)topquark rapidity. The results are further unfolded through a range of different techniques. For the dilepton channel an additional asymmetry, $A_{c}^{l e p}$ may be measured between the rapidity of the two leptons. $A_{c}^{l e p}$ does not require full reconstruction of the top-quark pair event and, as it is not fully correlated to $A_{c}$, provides extra information.

ATLAS and CMS measure $A_{c}$ and $A_{c}^{l e p}$ inclusively and differentially and find that all results are in agreement with the Standard Model (SM), as shown in Figure 1b. Both ATLAS and CMS measure differentially by the mass $\left(m_{t \bar{t}}\right)$, shown in Figures $2 \mathrm{a}$ and $2 \mathrm{~b}$, and a selection of other kinematic variables. A comparison of $A_{c}$ and $A_{c}^{l e p}$ is shown in Figure 2c probes BSM models but is unable to exclude with this level of precision. CMS also report [6] an inclusive measurement of $A_{c}$ in the $\ell+$ jets channel using a template method which achieves reduced uncertainties.

ATLAS measures [7] $A_{c}$ using reconstruction techniques specifically designed for the decay topology of highly boosted top quarks, a phase space that is enriched with the $q \bar{q} \rightarrow t \bar{t}$ process and thus more sensitive to BSM physics. The results are measured inclusively and differentially by $m_{t \bar{t}}$, are found to be consistent with the SM and provide a constraints on certain BSM models, some of which predict a very sizable $A_{c}$ at large $m_{t \bar{t}}$.

The $A_{F B}$ results are used in conjunction with the $A_{c}$ results to constrain BSM models for different ranges of $m_{t \bar{t}}$, as shown in Figure 3 The uncertainties on the measurements are too large to rule out Beyond the Standard Model (BSM) theories. 


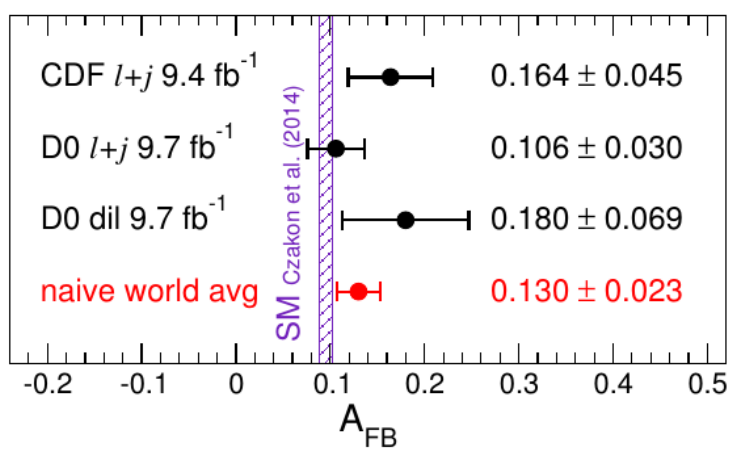

(a) Tevatron $A_{F B}$ summary.

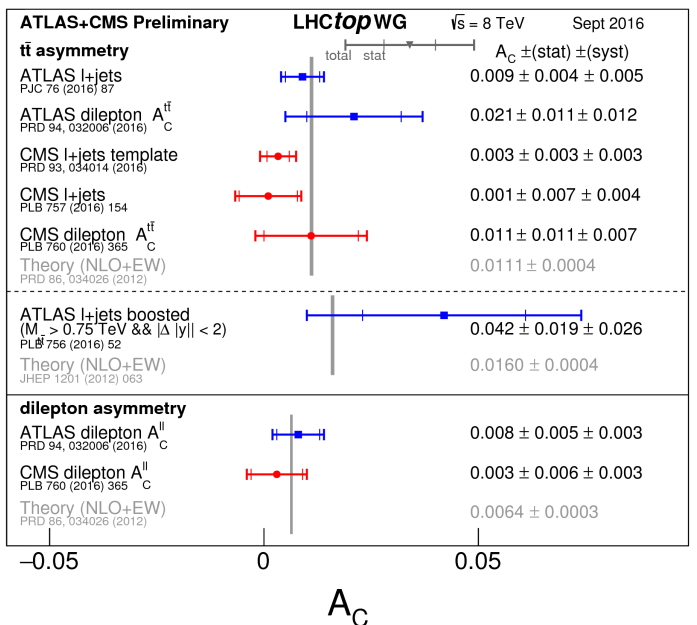

(b) LHC $A_{c}$ and $A_{c}^{l e p}$ summary.

Figure 1: Summary of inclusive asymmetry results.

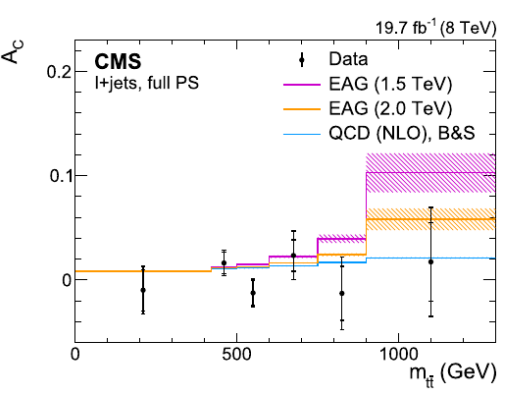

(a) CMS $\ell+$ jets $A_{c}$ Vs $m_{t \bar{t}}$

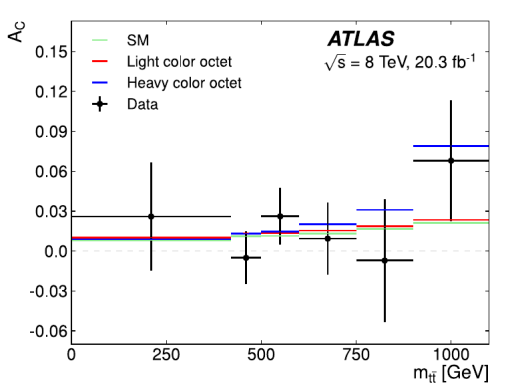

(b) ATLAS $\ell+$ jets $A_{c}$ Vs $m_{t \bar{t}}$

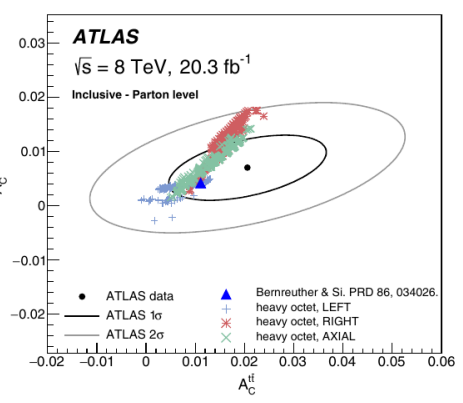

(c) ATLAS dilepton $A_{c}$ Vs $A_{c}^{l e p}$

Figure 2: Selected charge asymmetry figures.

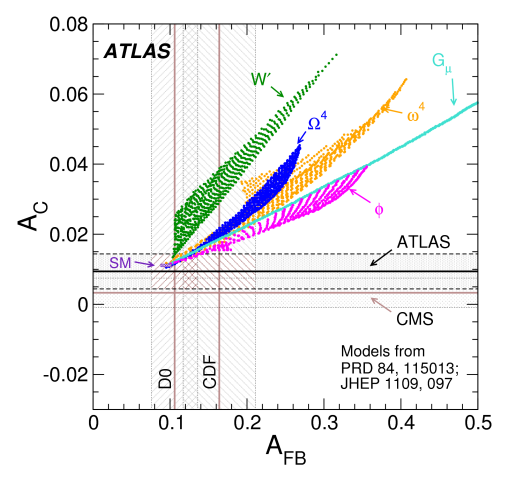

(a) Resolved

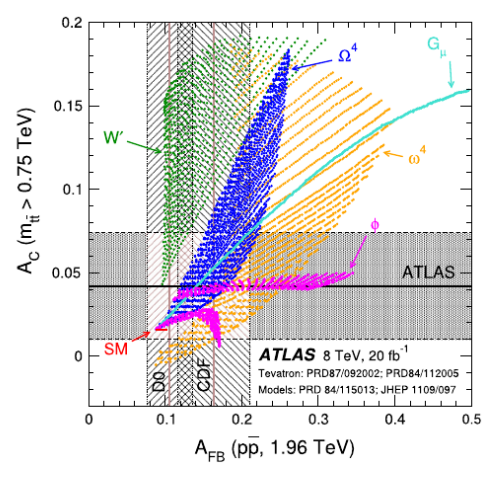

(b) $m_{t \bar{t}}>0.75 \mathrm{TeV}$

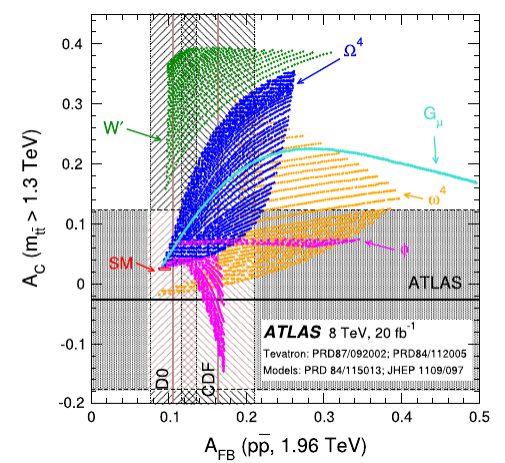

(c) $m_{t \bar{t}}>1.3 \mathrm{TeV}$

Figure 3: SM and BSM predictions for $A_{F B}$ Vs $A_{c}$ with Tevatron and LHC results and predictions. Shown for three different ranges of $m_{t \bar{t}}$. 


\section{Measurements of charge and CP asymmetries using top-pair events at ATLAS}

The abundance of top quarks produced at the LHC makes it possible to perform measurements [8] of CP asymmetries in heavy-flavour mixing and decay from top-quark decay products. All existing analyses of CP violation in $B$-meson decays rely on a resonant production of $b \bar{b}$ pairs or hadroproduction. The unique aspect presented in this paper is the method by which the charge of the $b$-quark is determined, both at production and at decay. The top quark decays before hadronisation, predominantly via $t \rightarrow W b$. In the case where the $W$-boson decays leptonically, the charge of the lepton determines the charge of the produced $b$-quark. The $b$-quark hadronises and in the case that the resulting $b$-hadron decays semileptonically to a muon, the charge of the soft muon determines the $b$-quark charge at decay. A Soft Muon Tagging (SMT) algorithm was developed to identify such muons. Experimental ambiguity in determining the charge of the produced $b$-quark arises in $t \bar{t}$ pair production events when establishing if an SMT muon originated from the same- or different-top quark as the leptonically decaying $W$-boson, as illustrated in Figures $4 \mathrm{a}$ and $4 \mathrm{~b}$.

Experimentally observable charge asymmetries are formed by considering the relative difference in the probability for an initial $b$ - or $\bar{b}$-quark to decay via either a positively or negatively charged SMT muon. Let $N^{\alpha \beta}$ represent the number of SMT muons observed with a charge $\beta$ in conjunction with a $W$-boson lepton of charge $\alpha$, where $\alpha, \beta= \pm 1$. In the case that an SMT muon is estimated to have originated from the different top-quark to the $W$-boson lepton, the sign of the $W$-boson lepton, $\alpha$, is flipped in order to consistently represent the charge of the $b$-quark at production in both scenarios. In the case of events where both $b$-hadrons decay semileptonically and are both experimentally tagged, the event contributes twice to the asymmetries. Observable sameand opposite-sign charge asymmetries are given by:

$$
A^{\mathrm{ss}}=\frac{\left(\frac{N^{++}}{N^{+}}-\frac{N^{--}}{N^{-}}\right)}{\left(\frac{N^{++}}{N^{+}}+\frac{N^{--}}{N^{-}}\right)}, \quad A^{\mathrm{os}}=\frac{\left(\frac{N^{+-}}{N^{+}}-\frac{N^{-+}}{N^{-}}\right)}{\left(\frac{N^{+-}}{N^{+}}+\frac{N^{-+}}{N^{-}}\right)} .
$$

The data are separated into same- and different-top-like SMT muons by a kinematic likelihood fitter with a misassignment probability of $21 \%$. The yield of same-top-like SMT muons for the $e+$ jets channel is shown in Figure 4c. The observed data are unfolded to a well-defined fiducial region and the charge asymmetries of Eq. 3.1 are measured. The charge asymmetries are related to the $\mathrm{CP}$ asymmetries

$$
\begin{aligned}
& A^{\mathrm{ss}}=r_{b} A_{\mathrm{mix}}^{b \ell}+r_{c}\left(A_{\mathrm{dir}}^{b c}-A_{\mathrm{dir}}^{c \ell}\right)+r_{c \bar{c}}\left(A_{\mathrm{mix}}^{b c}-A_{\mathrm{dir}}^{c \ell}\right), \\
& A^{\mathrm{os}}=\widetilde{r}_{b} A_{\mathrm{dir}}^{b \ell}+\widetilde{r}_{c}\left(A_{\mathrm{mix}}^{b c}+A_{\mathrm{dir}}^{c \ell}\right)+\widetilde{r}_{c \bar{c}} A_{\mathrm{dir}}^{c \ell},
\end{aligned}
$$

where the decay-chain fractions, $r_{i}$ and $\widetilde{r}_{i}$, represent the relative rates of each decay-channel in the well-defined fiducial volume. Following a convention where $A_{\text {mix }}^{b \ell} \equiv A_{\text {mix }}^{b c} \equiv A_{\text {mix }}^{b}$ and considering each $\mathrm{CP}$ asymmetry in turn whilst setting all the others to zero, the results given in Table 1 are entirely consistent with the SM. The 2 standard deviation limits inferred by this analysis are stronger than the existing indirect limit on $A_{\mathrm{dir}}^{c \ell}$, and equivalent to the existing indirect limit on $A_{\mathrm{dir}}^{b \ell}$. 


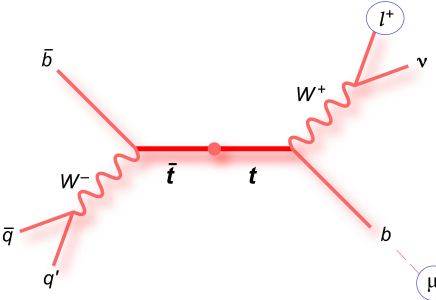

(a) Same-top SMT muon

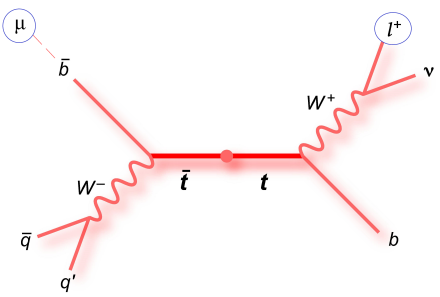

(b) Different-top SMT muon

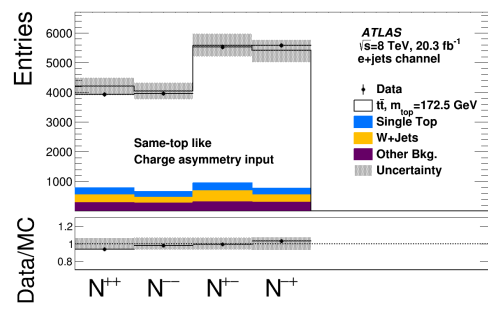

(c) Same-top-like charge-pairings distributions for $e+$ jets channel

Figure 4: Selected CP violation figures

\begin{tabular}{|c|c|c|c|c|c|c|}
\hline & \multicolumn{2}{|c|}{ Data $\left(10^{-2}\right)$} & \multicolumn{2}{|c|}{$\operatorname{MC}\left(10^{-2}\right)$} & Existing limits $(2 \sigma)\left(10^{-2}\right)$ & SM prediction $\left(10^{-2}\right)$ \\
\hline$A^{\mathrm{ss}}$ & -0.7 & \pm 0.8 & 0.05 & \pm 0.23 & - & $<10^{-2}$ \\
\hline$A^{\mathrm{os}}$ & 0.4 & \pm 0.5 & -0.03 & \pm 0.13 & - & $<10^{-2}$ \\
\hline$A_{\mathrm{mix}}^{b}$ & -2.5 & \pm 2.8 & 0.2 & \pm 0.7 & $<0.1$ & $<10^{-3}$ \\
\hline$A_{\mathrm{dir}}^{b \ell}$ & 0.5 & \pm 0.5 & -0.03 & \pm 0.14 & $<1.2$ & $<10^{-5}$ \\
\hline$A_{\mathrm{dir}}^{c \ell}$ & 1.0 & \pm 1.0 & -0.06 & \pm 0.25 & $<6.0$ & $<10^{-9}$ \\
\hline$A_{\mathrm{dir}}^{b c}$ & -1.0 & \pm 1.1 & 0.07 & \pm 0.29 & - & $<10^{-7}$ \\
\hline
\end{tabular}

Table 1: Comparison of measurements of charge asymmetries and constraints on $\mathrm{CP}$ asymmetries, with MC simulation (detailed in the text), existing experimental limits and SM predictions. The latter two columns represent upper limits on the absolute values $|A|$.

\section{T-odd triple product correlations using top-pair events at CMS}

CMS report [9] four CP-sensitive measurements, using $\ell+$ jets $t \bar{t}$ events, in which any nonzero asymmetry would probe for BSM models. The T-odd observables are measured using the linearly independent four-momentum vectors associated with the top-quark production and decay. The measured asymmetries shown no evidence for CP-violation effects in $t \bar{t}$ events and are in agreement with the SM.

\section{References}

[1] J. A. Aguilar-Saavedra et al., Rev. Mod. Phys. 87, 421 (2015) [arXiv:1406.1798 [hep-ph]].

[2] ATLAS Collaboration, Phys. Rev. D 94, no. 3, 032006 (2016) [arXiv:1604.05538 [hep-ex]].

[3] CMS Collaboration, Phys. Lett. B 760, 365 (2016) [arXiv:1603.06221 [hep-ex]].

[4] ATLAS Collaboration, Eur. Phys. J. C 76, no. 2, 87 (2016) [arXiv:1509.02358 [hep-ex]].

[5] CMS Collaboration, Phys. Lett. B 757, 154 (2016) [arXiv:1507.03119 [hep-ex]].

[6] CMS Collaboration, Phys. Rev. D 93, no. 3, 034014 (2016) [arXiv:1508.03862 [hep-ex]].

[7] ATLAS Collaboration, Phys. Lett. B 756, 52 (2016) [arXiv:1512.06092 [hep-ex]].

[8] ATLAS Collaboration, JHEP 1702 (2017) 071 [arXiv:1610.07869 [hep-ex]].

[9] CMS Collaboration, arXiv:1611.08931 [hep-ex]. 